PROCEEDINGS OF THE

AMERICAN MATHEMATICAL SOCIETY

Volume 137, Number 11, November 2009, Pages 3779-3786

S 0002-9939(09)10007-2

Article electronically published on June 9, 2009

\title{
AN INDEX THEOREM FOR TOEPLITZ OPERATORS ON THE QUARTER-PLANE
}

ADEL B. BADI

(Communicated by Marius Junge)

\begin{abstract}
We prove an index theorem for Toeplitz operators on the quarterplane using the index theory for generalized Toeplitz operators introduced by G. J. Murphy. To prove this index theorem we construct an indicial triple on the tensor product of two commutative symbol $\mathrm{C}^{*}$-algebras. We extend our results to matrices of Toeplitz operators on the quarter-plane.
\end{abstract}

\section{INTRODUCTION}

Define $L^{2}\left(\mathbb{T}^{2}\right)$ as the space of square-integrable functions relative to the normalized Lebesgue measure on $\mathbb{T}^{2}$, where $\mathbb{T}$ is the unit circle in the complex plane $\mathbb{C}$. The generalized Hardy space $H^{2}\left(\mathbb{T}^{2}\right)$ is defined as the set of $L^{2}$ functions on $\mathbb{T}^{2}$ whose Fourier transform vanishes off the set $\{(m, n) \mid m, n \geq 0\}$. Let $P_{\mathbb{T}^{2}}$ be the orthogonal projection of $L^{2}\left(\mathbb{T}^{2}\right)$ onto $H^{2}\left(\mathbb{T}^{2}\right)$. If $\varphi \in C\left(\mathbb{T}^{2}\right)$, then the Toeplitz operator on the quarter-plane with symbol $\varphi$ is $W_{\varphi}(f)=P_{\mathbb{T}^{2}}(\varphi f), f \in H^{2}\left(\mathbb{T}^{2}\right)$. These operators and the $\mathrm{C}^{*}$-algebra $\mathcal{T}_{\mathbb{T}^{2}}$ generated by them were studied by R. G. Douglas and R. Howe among others. They showed that there are Fredholm operators in $\mathcal{T}_{\mathbb{T}^{2}}$ of arbitrary integer index. However, they found that $W_{\varphi}$ is a Fredholm operator if and only if $\varphi=e^{\psi}$ for some $\psi \in C\left(\mathbb{T}^{2}\right)$ [2, Corollary of Theorem 1]. In this case the Fredholm index is zero. Thus, the classical Fredholm theory gives a trivial index for Toeplitz operators on the quarter-plane. Moreover, they found that their results do not hold for matrices of these operators [2, p. 214]. A similar situation appears in 11, where C. A. Berger and L. A. Coburn studied Toeplitz operators on the group $\mathbf{U}(2)$ of unitary $2 \times 2$ matrices. In [5], G. J. Murphy introduced a satisfactory index theorem for these operators using a generalization of Fredholm index theory he developed earlier in [4, and later he put his results in a general framework [6].

In this paper, we use results from 6 to obtain an index theorem for Toeplitz operators on the quarter-plane. In our index theorem, a Toeplitz operator $W_{\varphi}$ is a Fredholm operator (in a generalized sense) if and only if $\varphi$ is non-vanishing on $\mathbb{T}^{2}$ and the Fredholm index of $W_{\varphi}$ is $-\theta m-n$, where $\theta$ is a fixed irrational number and $m, n$ are the unique integers such that $\varphi=\left(z^{m} \otimes z^{n}\right) e^{\psi}$ for some $\psi \in C\left(\mathbb{T}^{2}\right)$ and $z$ is the function $\mathbb{T} \rightarrow \mathbb{C}, \lambda \mapsto \lambda$. Moreover, the Fredholm index of $W_{\varphi}$ is

Received by the editors February 21, 2008, and, in revised form, February 23, 2009.

2000 Mathematics Subject Classification. Primary 47B35; Secondary 47A53, 58B15.

Key words and phrases. Toeplitz operators on the quarter-plane, generalized Fredholm index, topological index, indicial triple.

(c)2009 American Mathematical Society Reverts to public domain 28 years from publication 
non-zero unless $\varphi=e^{\psi}$ for some $\psi \in C\left(\mathbb{T}^{2}\right)$. Finally, we have extended our results to matrices of Toeplitz operators on the quarter-plane.

In Section 2 we prove general results showing that we can define a topological index on the tensor product of two commutative symbol $\mathrm{C}^{*}$-algebras. Using these results, we proved our index theorem for Toeplitz operators on the quarter-plane in Section 3 ,

\section{INDEX THEORY FOR TENSOR PRODUCTS}

Suppose that $A$ is a $\mathrm{C}^{*}$-algebra and tr is a trace on $A$. We will denote the ideal of definition of tr by $\mathcal{M}_{\mathrm{tr}}$ and the closure of $\mathcal{M}_{\mathrm{tr}}$ by $\mathcal{K}_{\mathrm{tr}}$. If $a, b \in A$, then we denote the commutator $a b-b a$ by $[a, b]$. The closed commutator ideal of $A$ is denoted by $\operatorname{Com}(A)$. If $A$ is unital, then we denote the group of invertible elements in $A$ by $\operatorname{Inv}(A)$. All tensor products of $\mathrm{C}^{*}$-algebras in this paper are the spatial tensor products. The symbol $\odot$ denotes the algebraic tensor product.

Let $C$ and $\mathcal{L}$ be unital $\mathrm{C}^{*}$-algebras such that $\mathcal{L}$ contains $C$ as a unital $\mathrm{C}^{*}$ subalgebra. Suppose that $F$ is a self-adjoint unitary in $\mathcal{L}$ and that $\operatorname{tr}$ is a lower semicontinuous trace on $\mathcal{L}$. If the unital $*$-subalgebra $\left\{\varphi \in C \mid[F, \varphi] \in \mathcal{M}_{\text {tr }}\right\}$ is dense in $C$, then we call the triple $\Omega=(\mathcal{L}, F, \operatorname{tr})$ an indicial triple for $C$ [6. p. 266]. We usually denote the latter dense $*$-subalgebra by $C_{\Omega}$, and the commutator $[F, \varphi]$ will be denoted by $\mathrm{d} \varphi$, where $\varphi \in C$. To avoid trivialities, we will assume that any indicial triple in this paper satisfies the condition $P \notin \mathcal{M}_{\mathrm{tr}}$, where $P$ is the projection $(F+1) / 2$. This condition is equivalent to $P \notin \mathcal{K}_{\mathrm{tr}}$.

A topological index on a unital $\mathrm{C}^{*}$-algebra $A$ is defined as a locally constant mapping $\omega: \operatorname{Inv}(A) \rightarrow \mathbb{R}$ such that $\omega(\varphi \psi)=\omega(\varphi)+\omega(\psi)$, for all $\varphi, \psi \in \operatorname{Inv}(A)$. Let $\Omega=(\mathcal{L}, F, \operatorname{tr})$ be an indicial triple for the $\mathrm{C}^{*}$-algebra $C$. Then there exists a unique topological index $\omega$ on $C$ satisfying the equality $\omega(\varphi)=\frac{1}{2} \operatorname{tr}\left(\varphi^{-1} \mathrm{~d} \varphi\right)$, for all $\varphi \in \operatorname{Inv}\left(C_{\Omega}\right)$; see [6, Theorem 2.6].

Define $P_{\mathbb{T}}$ as the orthogonal projection of the space $L^{2}(\mathbb{T})$ onto the classical Hardy space $H^{2}(\mathbb{T})$. Let $\mathcal{L}_{\mathbb{T}}$ be the unital $\mathrm{C}^{*}$-subalgebra of $\mathbf{B}\left(L^{2}(\mathbb{T})\right)$ generated by $C(\mathbb{T})$ and $P_{\mathbb{T}}$, where $C(\mathbb{T})$ is identified with its corresponding $\mathrm{C}^{*}$-algebra of multiplication operators on $L^{2}(\mathbb{T})$. If we define the trace $\operatorname{tr}_{\mathbb{T}}$ as the restriction of the canonical trace on $\mathbf{B}\left(L^{2}(\mathbb{T})\right)$ to $\mathcal{L}_{\mathbb{T}}$ and we define $F_{\mathbb{T}}$ as the self-adjoint unitary $2 P_{\mathbb{T}}-1$, then, by [6. Example 2.7], $\Omega_{\mathbb{T}}=\left(\mathcal{L}_{\mathbb{T}}, F_{\mathbb{T}}, \operatorname{tr}_{\mathbb{T}}\right)$ is an indicial triple for $C(\mathbb{T})$. If $\varphi \in \operatorname{Inv}(C(\mathbb{T}))$, then $\omega_{\mathbb{T}}(\varphi)=\operatorname{wn}(\varphi)$, where $\omega_{\mathbb{T}}$ is the topological index related to the indicial triple $\Omega_{\mathbb{T}}$ and $\operatorname{wn}(\varphi)$ is the winding number of $\varphi$.

We want to construct an indicial triple for $C\left(\mathbb{T}^{2}\right)=C(\mathbb{T}) \otimes C(\mathbb{T})$ to obtain an index theorem for Toeplitz operators on the quarter-plane. The equality $L^{2}\left(\mathbb{T}^{2}\right)=$ $L^{2}(\mathbb{T}) \otimes L^{2}(\mathbb{T})$ implies $P_{\mathbb{T}^{2}}=P_{\mathbb{T}} \otimes P_{\mathbb{T}}$ and $\mathcal{L}_{\mathbb{T}} \otimes \mathcal{L}_{\mathbb{T}} \subseteq \mathbf{B}\left(L^{2}\left(\mathbb{T}^{2}\right)\right)$. Let $F_{\mathbb{T}^{2}}=2 P_{\mathbb{T}^{2}}-1$ and let $\mathcal{L}_{\mathbb{T}^{2}}=\mathcal{L}_{\mathbb{T}} \otimes \mathcal{L}_{\mathbb{T}}$. Then $C\left(\mathbb{T}^{2}\right) \subseteq \mathcal{L}_{\mathbb{T}^{2}}$ and $F_{\mathbb{T}^{2}} \in \mathcal{L}_{\mathbb{T}^{2}}$.

Let $\operatorname{tr}_{0}$ be the restriction of the canonical trace on $\mathbf{B}\left(L^{2}\left(\mathbb{T}^{2}\right)\right)$ to $\mathcal{L}_{\mathbb{T}^{2}}$. Then $\mathcal{M}_{\operatorname{tr}_{\mathbb{T}}} \odot \mathcal{M}_{\operatorname{tr}_{\mathbb{T}}} \subseteq \mathcal{M}_{\operatorname{tr}_{0}}, \mathcal{K}_{\operatorname{tr}_{\mathbb{T}}} \otimes \mathcal{K}_{\operatorname{tr}_{\mathbb{T}}}=\mathcal{K}_{\operatorname{tr}_{0}}$ and

$$
\operatorname{tr}_{0}(a \otimes b)=\operatorname{tr}_{\mathbb{T}}(a) \operatorname{tr}_{\mathbb{T}}(b) \quad\left(a, b \in \mathcal{M}_{\mathrm{tr}_{\mathbb{T}}}\right) .
$$

Moreover, $\operatorname{tr}_{0}$ is the only lower semicontinuous trace with these properties. Hence, we can view the trace $\operatorname{tr}_{0}$ as the tensor product $\operatorname{tr}_{\mathbb{T}} \otimes \operatorname{tr}_{\mathbb{T}}$ of the trace $\operatorname{tr}_{\mathbb{T}}$ with itself. The trace $\operatorname{tr}_{0}$ with $\mathcal{L}_{\mathbb{T}^{2}}$ and $F_{\mathbb{T}^{2}}$ does not define an indicial triple for $C\left(\mathbb{T}^{2}\right)$. To see this, suppose that $\left(\mathcal{L}_{\mathbb{T}^{2}}, F_{\mathbb{T}^{2}}, \operatorname{tr}_{0}\right)$ is an indicial triple. Then the unital $*$-subalgebra $\left\{\varphi \in C\left(\mathbb{T}^{2}\right) \mid \mathrm{d} \varphi \in \mathcal{M}_{\text {tro }_{0}}\right\}$ is dense in $C\left(\mathbb{T}^{2}\right)$. Consider the function $z \otimes 1 \in C\left(\mathbb{T}^{2}\right)$. 
There is a sequence $\left(\varphi_{m}\right)_{m=1}^{\infty}$ in $C\left(\mathbb{T}^{2}\right)$ such that $\mathrm{d} \varphi_{m} \in \mathcal{M}_{\operatorname{tr}_{0}}$ and $\varphi_{m} \rightarrow z \otimes 1$. This implies

$$
2\left[P_{\mathbb{T}}, z\right] \otimes P_{\mathbb{T}}=\mathrm{d}(z \otimes 1) \in \mathcal{K}_{\operatorname{tr}_{0}} \subseteq \mathbf{K}\left(L^{2}\left(\mathbb{T}^{2}\right)\right),
$$

a contradiction.

It is obvious now that the main difficulty in the construction of an indicial triple for $C\left(\mathbb{T}^{2}\right)$ is finding a suitable trace. Similarly, the construction of the trace for defining a generalized Fredholm index for Toeplitz operators on the group $\mathbf{U}(2)$ is the main result in 5 .

Let $A, B$ be $\mathrm{C}^{*}$-algebras and tr be a lower semicontinuous trace on $A$ and let $\tau$ be a character on $B$. Then we define the lower semicontinuous trace $\operatorname{tr} \otimes \tau$ on $A \otimes B$ by the equality $(\operatorname{tr} \otimes \tau)(a)=\operatorname{tr}((\iota \otimes \tau)(a)), a \in(A \otimes B)^{+}$.

Lemma 2.1. Suppose that $A, B$ are $\mathrm{C}^{*}$-algebras. Let $\operatorname{tr}_{A}, \operatorname{tr}_{B}$ be lower semicontinuous traces and let $\tau_{A}, \tau_{B}$ be characters on $A, B$, respectively. If $a \in A$ and $b \in \mathcal{M}_{\operatorname{tr}_{B}} \cap \operatorname{ker}\left(\tau_{B}\right)$, then $a \otimes b$ belongs to the definition ideal of the trace $\theta_{1} \operatorname{tr}_{A} \otimes \tau_{B}+\theta_{2} \tau_{A} \otimes \operatorname{tr}_{B}$, where $\theta_{1}, \theta_{2}$ are fixed positive numbers. Moreover,

$$
\left(\operatorname{tr}_{A} \otimes \tau_{B}\right)(a \otimes b)=0 .
$$

Proof. We have $|b| \in \operatorname{ker}\left(\tau_{B}\right)$ since $b$ belongs to the $\mathrm{C}^{*}$-subalgebra $\operatorname{ker}\left(\tau_{B}\right)$ of $B$. Note that $|a \otimes b|=|a| \otimes|b|$. Hence,

$$
\begin{aligned}
\left(\operatorname{tr}_{A} \otimes \tau_{B}\right)(|a \otimes b|) & =\operatorname{tr}_{A}\left(\left(\iota \otimes \tau_{B}\right)(|a| \otimes|b|)\right) \\
& =\operatorname{tr}_{A}\left(\tau_{B}(|b|)|a|\right)=\operatorname{tr}_{A}(0 \cdot|a|)=0 .
\end{aligned}
$$

Thus, $|a \otimes b| \in \mathcal{M}_{\operatorname{tr}_{A} \otimes \tau_{B}}^{+}$.

By [7, Prop. A1(b)], we have $\left|\left(\operatorname{tr}_{A} \otimes \tau_{B}\right)(a \otimes b)\right| \leq\left(\operatorname{tr}_{A} \otimes \tau_{B}\right)(|a \otimes b|)$. Hence, $\left(\operatorname{tr}_{A} \otimes \tau_{B}\right)(a \otimes b)=0$. By [7, Prop. A1(a)], $|b| \in \mathcal{M}_{\operatorname{tr}_{B}}$ and it follows immediately that $|a \otimes b|=|a| \otimes|b| \in \mathcal{M}_{\tau_{A} \otimes \operatorname{tr}_{B}}^{+}$. Thus, $|a \otimes b|$ belongs to the definition ideal of the $\operatorname{trace} \theta_{1} \operatorname{tr}_{A} \otimes \tau_{B}+\theta_{2} \tau_{A} \otimes \operatorname{tr}_{B}$ and again by [7, Prop. A1(a)], $a \otimes b$ belongs to this ideal too.

The following theorem describes the construction of the trace on tensor products, and it gives a simple formula to calculate the index for elementary tensors of invertible elements.

Theorem 2.2. Let $\Omega_{i}=\left(\mathcal{L}_{i}, F_{i}, \operatorname{tr}_{i}\right)$ be an indicial triple for the $\mathrm{C}^{*}$-algebra $C_{i}$ and let $P_{i}=\left(F_{i}+1\right) / 2$, where $i=1,2$. If there exists a character $\tau_{i}$ on $\mathcal{L}_{i}$ such that $\tau_{i}\left(P_{i}\right) \neq 0$, then $\Omega=(\mathcal{L}, F, \operatorname{tr})$ is an indicial triple for $C=C_{1} \otimes C_{2}$, where $\mathcal{L}=\mathcal{L}_{1} \otimes \mathcal{L}_{2}, F=2\left(P_{1} \otimes P_{2}\right)-1$ and the trace $\operatorname{tr}: \mathcal{L}^{+} \rightarrow[0,+\infty]$ is defined as

$$
\operatorname{tr}(a)=\theta_{1}\left(\operatorname{tr}_{1} \otimes \tau_{2}\right)(a)+\theta_{2}\left(\tau_{1} \otimes \operatorname{tr}_{2}\right)(a) \quad\left(a \in \mathcal{L}^{+}\right),
$$

where $\theta_{1}, \theta_{2}$ are fixed positive numbers. Moreover,

$$
\omega\left(\varphi_{1} \otimes \varphi_{2}\right)=\theta_{1} \omega_{1}\left(\varphi_{1}\right)+\theta_{2} \omega_{2}\left(\varphi_{2}\right)
$$

for all $\varphi_{1} \in \operatorname{Inv}\left(C_{1}\right), \varphi_{2} \in \operatorname{Inv}\left(C_{2}\right)$, where $\omega_{1}, \omega_{2}, \omega$ are the topological indices related to the indicial triples $\Omega_{1}, \Omega_{2}, \Omega$, respectively.

Proof. We show that $\mathrm{d} \varphi \in \mathcal{M}_{\mathrm{tr}}$ for every $\varphi$ in the unital dense *-subalgebra $C_{\Omega_{1}} \odot C_{\Omega_{2}}$ of $C$. To do this, it is enough to show that $\mathrm{d}\left(\varphi_{1} \otimes \varphi_{2}\right) \in \mathcal{M}_{\mathrm{tr}}$, for all $\varphi_{1} \in C_{\Omega_{1}}, \varphi_{2} \in C_{\Omega_{2}}$. Note that

$$
\mathrm{d}\left(\varphi_{1} \otimes \varphi_{2}\right)=2\left(\left[P_{1}, \varphi_{1}\right] \otimes P_{2} \varphi_{2}+\varphi_{1} P_{1} \otimes\left[P_{2}, \varphi_{2}\right]\right) .
$$


By Lemma 2.1. $\left[P_{1}, \varphi_{1}\right] \otimes P_{2} \varphi_{2}$ and $\varphi_{1} P_{1} \otimes\left[P_{2}, \varphi_{2}\right]$ belong to the definition ideal of the trace $\operatorname{tr}=\theta_{1} \operatorname{tr}_{1} \otimes \tau_{2}+\theta_{2} \tau_{1} \otimes \operatorname{tr}_{2}$. Hence, $\mathrm{d}\left(\varphi_{1} \otimes \varphi_{2}\right) \in \mathcal{M}_{\mathrm{tr}}$. It follows immediately now that $\Omega=(\mathcal{L}, F, \operatorname{tr})$ is an indicial triple for $C$.

Now we prove the equality $\omega\left(\varphi_{1} \otimes \varphi_{2}\right)=\theta_{1} \omega_{1}\left(\varphi_{1}\right)+\theta_{2} \omega_{2}\left(\varphi_{2}\right)$. By continuity, it is sufficient to prove it for $\varphi_{1} \in \operatorname{Inv}\left(C_{\Omega_{1}}\right), \varphi_{2} \in \operatorname{Inv}\left(C_{\Omega_{2}}\right)$. Note that

$$
\begin{aligned}
\omega\left(\varphi_{1} \otimes \varphi_{2}\right) & =\frac{1}{2} \operatorname{tr}\left(\left(\varphi_{1} \otimes \varphi_{2}\right)^{-1} \mathrm{~d}\left(\varphi_{1} \otimes \varphi_{2}\right)\right) \\
& =\operatorname{tr}\left(\varphi_{1}^{-1}\left[P_{1}, \varphi_{1}\right] \otimes \varphi_{2}^{-1} P_{2} \varphi_{2}+P_{1} \otimes \varphi_{2}^{-1}\left[P_{2}, \varphi_{2}\right]\right) .
\end{aligned}
$$

We calculate each item separately. It is obvious that $\varphi_{1}^{-1}\left[P_{1}, \varphi_{1}\right] \in \operatorname{ker}\left(\tau_{1}\right)$ and $\varphi_{2}^{-1}\left[P_{2}, \varphi_{2}\right] \in \operatorname{ker}\left(\tau_{2}\right)$. Hence, by Lemma 2.1, we have

$$
\left(\operatorname{tr}_{1} \otimes \tau_{2}\right)\left(P_{1} \otimes \varphi_{2}^{-1}\left[P_{2}, \varphi_{2}\right]\right)=\left(\tau_{1} \otimes \operatorname{tr}_{2}\right)\left(\varphi_{1}^{-1}\left[P_{1}, \varphi_{1}\right] \otimes \varphi_{2}^{-1} P_{2} \varphi_{2}\right)=0 .
$$

By Lemma 2.1, $P_{1} \otimes \varphi_{2}^{-1}\left[P_{2}, \varphi_{2}\right]$ belongs to the definition ideal of the trace $\tau_{1} \otimes \operatorname{tr}_{2}$ and

$$
\left(\tau_{1} \otimes \operatorname{tr}_{2}\right)\left(P_{1} \otimes \varphi_{2}^{-1}\left[P_{2}, \varphi_{2}\right]\right)=\tau_{1}\left(P_{1}\right) \operatorname{tr}_{2}\left(\varphi_{2}^{-1}\left[P_{2}, \varphi_{2}\right]\right)=\omega_{2}\left(\varphi_{2}\right),
$$

since $\tau_{1}\left(P_{1}\right)=1$. Similarly, $\left(\operatorname{tr}_{1} \otimes \tau_{2}\right)\left(\varphi_{1}^{-1}\left[P_{1}, \varphi_{1}\right] \otimes \varphi_{2}^{-1} P_{2} \varphi_{2}\right)=\omega_{1}\left(\varphi_{1}\right)$.

Hence, $\omega\left(\varphi_{1} \otimes \varphi_{2}\right)=\theta_{1} \omega_{1}\left(\varphi_{1}\right)+\theta_{2} \omega_{2}\left(\varphi_{2}\right)$.

In this theorem, the value of the index at elementary invertible tensors is independent of the choice of the characters. The construction of the indicial triple in Theorem 2.2 depends on the existence of the characters $\tau_{1}, \tau_{2}$. We need the following lemma to show that these characters do exist under general conditions.

Lemma 2.3. Let $\Omega=(\mathcal{L}, F, \operatorname{tr})$ be an indicial triple for the commutative $\mathrm{C}^{*}$-algebra $C$ and let $P=(F+1) / 2$. If $\mathcal{L}=\mathrm{C}^{*}(C, P)$ (the $\mathrm{C}^{*}$-algebra generated by $C$ and $P$ ), then $\operatorname{Com}(\mathcal{L}) \subseteq \mathcal{K}_{\text {tr }}$.

Proof. Let $C_{\Omega}=\left\{\varphi \in C \mid \mathrm{d} \varphi=2[P, \varphi] \in \mathcal{M}_{\mathrm{tr}}\right\}$. If $\varphi \in C$, then, by the density of $C_{\Omega},[P, \varphi] \in \mathcal{K}_{\mathrm{tr}}$. Denote by $\mathcal{S}$ the set of all elements of $\mathcal{L}$ of the form

$$
a=\varphi_{0} P \varphi_{1} P \varphi_{2} \cdots \varphi_{n-1} P \varphi_{n},
$$

where $n$ is a non-negative integer and $\varphi_{i} \in C$, for all $0 \leq i \leq n$. Hence, for such an element $a \in \mathcal{S}$, we have

$$
\begin{aligned}
{[a, P]=} & {\left[\varphi_{0}, P\right] P \varphi_{1} P \varphi_{2} \cdots \varphi_{n-1} P \varphi_{n}+\varphi_{0} P\left[\varphi_{1}, P\right] P \varphi_{2} \cdots \varphi_{n-1} P \varphi_{n}+} \\
& \cdots+\varphi_{0} P \varphi_{1} P \varphi_{2} \cdots \varphi_{n-1} P\left[\varphi_{n}, P\right] .
\end{aligned}
$$

Moreover, if $\varphi \in C$, then

$$
\begin{aligned}
{[a, \varphi]=} & \varphi_{0}[P, \varphi] \varphi_{1} P \varphi_{2} \cdots \varphi_{n-1} P \varphi_{n}+\varphi_{0} P \varphi_{1}[P, \varphi] \varphi_{2} \cdots \varphi_{n-1} P \varphi_{n}+ \\
& \cdots+\varphi_{0} P \varphi_{1} P \varphi_{2} \cdots \varphi_{n-1}[P, \varphi] \varphi_{n} .
\end{aligned}
$$

Note that we need the commutativity of $C$ to show that the latter equality holds. Since $\mathcal{K}_{\text {tr }}$ is an ideal, we have $[a, P],[a, \varphi] \in \mathcal{K}_{\text {tr }}$ for any $a \in \mathcal{S}, \varphi \in C$. Now, if $b \in \mathcal{S}$, then

$$
\begin{aligned}
{[a, b]=} & {\left[\varphi_{0}, b\right] P \varphi_{1} P \varphi_{2} \cdots \varphi_{n-1} P \varphi_{n}+\varphi_{0}[P, b] \varphi_{1} P \varphi_{2} \cdots \varphi_{n-1} P \varphi_{n} } \\
& +\varphi_{0} P\left[\varphi_{1}, b\right] P \psi_{2} \cdots \varphi_{n-1} P \varphi_{n}+\cdots+\varphi_{0} P \varphi_{1} P \varphi_{2} \cdots \varphi_{n-1} P\left[\varphi_{n}, b\right] .
\end{aligned}
$$

Thus, $[a, b] \in \mathcal{K}_{\mathrm{tr}}$, for all $a, b \in \mathcal{S}$. Denote by $\mathcal{D}$ the set of all the sums $\sum_{i=1}^{m} a_{i}$, where $a_{i} \in \mathcal{S}, 1 \leq i \leq m$, for some positive integer $m$. Note that $\mathcal{D}$ is a unital *-subalgebra of $\mathcal{L}$ and $C \cup\{P\} \subseteq \mathcal{D} \subseteq \mathcal{L}$. Hence, $\mathcal{D}$ is dense in $\mathcal{L}$. Let $a, b \in \mathcal{D}$. 
Then $a=\sum_{i=1}^{m} a_{i}$ and $b=\sum_{j=1}^{n} b_{j}$, where $a_{i}, b_{j} \in \mathcal{S}$ for $1 \leq i \leq m$ and $1 \leq j \leq n$, for some positive integers $m, n$. Thus, $[a, b]=\sum_{i, j=1}^{m, n}\left[a_{i}, b_{j}\right] \in \mathcal{K}_{\mathrm{tr}}$. Hence, by the density of $\mathcal{D}$, we can easily show that $[a, b] \in \mathcal{K}_{\text {tr }}$, for any pair of elements $a, b$ in $\mathcal{L}$. This implies that the closed commutator ideal of $\mathcal{L}$ is a subset of $\mathcal{K}_{\text {tr }}$.

Theorem 2.4. Let $\Omega=(\mathcal{L}, F, \operatorname{tr})$ be an indicial triple for the commutative $\mathrm{C}^{*}-$ algebra $C$ such that $\mathcal{L}=\mathrm{C}^{*}(C, P)$, where $P=(F+1) / 2$. Then there exists a character $\tau$ on $\mathcal{L}$ such that $\tau(P) \neq 0$.

\section{An indeX theorem For TOePlitz operators on the QUARTER-Plane}

Let $\Omega_{\mathbb{T}}=\left(\mathcal{L}_{\mathbb{T}}, F_{\mathbb{T}}, \operatorname{tr}_{\mathbb{T}}\right)$ be the indicial triple for $C(\mathbb{T})$ defined in Section 2 and let $\omega_{\mathbb{T}}$ be the unique topological index related to $\Omega_{\mathbb{T}}$. By Theorem 2.4 there exists a character $\tau$ on $\mathcal{L}_{\mathbb{T}}$ such that $\tau\left(P_{\mathbb{T}}\right) \neq 0$. As in the previous section, we set $\mathcal{L}_{\mathbb{T}^{2}}=\mathcal{L}_{\mathbb{T}} \otimes \mathcal{L}_{\mathbb{T}}, F_{\mathbb{T}^{2}}=2 P_{\mathbb{T}^{2}}-1$. By Theorem 2.2 , the triple $\Omega_{1}=\left(\mathcal{L}_{\mathbb{T}^{2}}, F_{\mathbb{T}^{2}}, \operatorname{tr}_{1}\right)$ is an indicial triple for the $\mathrm{C}^{*}$-algebra $C\left(\mathbb{T}^{2}\right)$, where $\operatorname{tr}_{1}$ is the lower semicontinuous trace defined as

$$
\operatorname{tr}_{1}: \mathcal{L}_{\mathbb{T}^{2}}^{+} \longrightarrow[0, \infty], \quad \operatorname{tr}_{1}(a)=\left(\tau \otimes \operatorname{tr}_{\mathbb{T}}\right)(a)+\theta\left(\operatorname{tr}_{\mathbb{T}} \otimes \tau\right)(a) \quad\left(a \in \mathcal{L}_{\mathbb{T}^{2}}^{+}\right),
$$

where $\theta$ is a fixed positive irrational number. We will see later in Corollary 3.6 the reason for making such a choice for $\theta$. To be able to prove Theorem 3.5 below, we will redefine the trace $\operatorname{tr}_{1}$. We need the following lemma to do this.

Lemma 3.1. In the $\mathrm{C}^{*}$-algebra $\mathcal{L}_{\mathbb{T}}$, we have $\mathcal{K}_{\operatorname{tr}_{\mathbb{T}}}=\mathbf{K}\left(L^{2}(\mathbb{T})\right)=\operatorname{Com}\left(\mathcal{L}_{\mathbb{T}}\right)$.

Proof. By the proof of Theorem 3.3 in [6], we have $\mathcal{K}_{\mathrm{tr}_{\mathbb{T}}}=\mathbf{K}\left(L^{2}(\mathbb{T})\right)$.

By Lemma 2.3, $\operatorname{Com}\left(\mathcal{L}_{\mathbb{T}}\right) \subseteq \mathcal{K}_{\mathrm{tr}_{\mathbb{T}}}$. The ideal $\operatorname{Com}\left(\mathcal{L}_{\mathbb{T}}\right)$ contains the rank one operator $\left[P_{\mathbb{T}}, z\right]$; therefore it contains all rank one operators. Hence, by $[3$, Theorem 2.4.6], $\operatorname{Com}\left(\mathcal{L}_{\mathbb{T}}\right)$ contains the ideal of finite rank operators, and this implies that $\mathbf{K}\left(L^{2}(\mathbb{T})\right) \subseteq \operatorname{Com}\left(\mathcal{L}_{\mathbb{T}}\right)$. Hence, $\operatorname{Com}\left(\mathcal{L}_{\mathbb{T}}\right)=\mathcal{K}_{\text {tr }_{\mathbb{T}}}$.

The closed ideal

$$
I=\mathcal{L}_{\mathbb{T}} \otimes \mathcal{K}_{\operatorname{tr}_{\mathbb{T}}}+\mathcal{K}_{\operatorname{tr}_{\mathbb{T}}} \otimes \mathcal{L}_{\mathbb{T}}=\left\{a+b \mid a \in \mathcal{L}_{\mathbb{T}} \otimes \mathcal{K}_{\operatorname{tr}_{\mathbb{T}}}, b \in \mathcal{K}_{\operatorname{tr}_{\mathbb{T}}} \otimes \mathcal{L}_{\mathbb{T}}\right\}
$$

is contained in $\mathcal{K}_{\mathrm{tr}_{1}}$. To see this let $a \in \mathcal{L}_{\mathbb{T}}, b \in \mathcal{M}_{\mathrm{tr}_{\mathbb{T}}}$. Then $|b| \in \mathcal{M}_{\mathrm{tr}_{\mathbb{T}}}^{+}$, by [7, Proposition A1(a)]. By Lemma 3.1, $|b| \in \mathcal{K}_{\text {tr }_{\mathbb{T}}}=\operatorname{Com}\left(\mathcal{L}_{\mathbb{T}}\right)$. Thus, $\tau(|b|)=0$. Hence, it follows that $|a \otimes b|=|a| \otimes|b| \in \mathcal{M}_{\mathrm{tr}_{1}}^{+}$. Again by [7, Proposition A1(a)], $a \otimes b \in \mathcal{M}_{\mathrm{tr}_{1}}$. Thus, $\mathcal{L}_{\mathbb{T}} \otimes \mathcal{K}_{\mathrm{tr}_{\mathbb{T}}} \subseteq \mathcal{K}_{\mathrm{tr}_{1}}$. Similarly, $\mathcal{K}_{\mathrm{tr}_{\mathbb{T}}} \otimes \mathcal{L}_{\mathbb{T}} \subseteq \mathcal{K}_{\mathrm{tr}_{1}}$. We define the lower semicontinuous trace $\operatorname{tr}_{\mathbb{T}^{2}}: \mathcal{L}_{\mathbb{T}^{2}}^{+} \rightarrow[0,+\infty]$ by the equality

$$
\operatorname{tr}_{\mathbb{T}^{2}}(a)= \begin{cases}\operatorname{tr}_{1}(a), & \text { if } a \in I^{+}, \\ +\infty, & \text { if } a \in \mathcal{L}_{\mathbb{T}^{2}}^{+} \backslash I^{+}\end{cases}
$$

Moreover, $\mathcal{K}_{\operatorname{tr}_{\mathbb{T} 2}}=I$. If $\varphi_{1}, \varphi_{2} \in C_{\Omega_{\mathbb{T}}}$, then

$$
\mathrm{d}\left(\varphi_{1} \otimes \varphi_{2}\right)=2\left(\left[P_{\mathbb{T}}, \varphi_{1}\right] \otimes P_{\mathbb{T}} \varphi_{2}+\varphi_{1} P_{\mathbb{T}} \otimes\left[P_{\mathbb{T}}, \varphi_{2}\right]\right) \in \mathcal{M}_{\operatorname{tr}_{1}} \cap I=\mathcal{M}_{\mathrm{tr}_{\mathbb{T} 2}} .
$$

Hence, the triple $\Omega_{\mathbb{T}^{2}}=\left(\mathcal{L}_{\mathbb{T}^{2}}, F_{\mathbb{T}^{2}}, \operatorname{tr}_{\mathbb{T}^{2}}\right)$ is an indicial triple for $C\left(\mathbb{T}^{2}\right)$. Denote by $\omega_{\mathbb{T}^{2}}$ the unique topological index related to $\Omega_{\mathbb{T}^{2}}$.

Proposition 3.2. If $\varphi \in \operatorname{Inv}\left(C\left(\mathbb{T}^{2}\right)\right)$, then $\omega_{\mathbb{T}^{2}}(\varphi)=\theta m+n$, where $m, n$ are the unique integers such that $\varphi=\left(z^{m} \otimes z^{n}\right) e^{\psi}$, for some $\psi \in C\left(\mathbb{T}^{2}\right)$. 
Proof. Let $\varphi \in \operatorname{Inv}\left(C\left(\mathbb{T}^{2}\right)\right)$. By a theorem of van Kampen [9], there exists a unique character $\chi$ on the group $\mathbb{T}^{2}$ and a continuous function $\psi$ on $\mathbb{T}^{2}$ such that $\varphi=\chi e^{\psi}$. By [8. Theorem 2.2.2], $\chi=z^{m} \otimes z^{n}$, for a unique pair of integers $m, n \in \mathbb{Z}$. Thus, we have $\varphi=\left(z^{m} \otimes z^{n}\right) e^{\psi}$. To calculate the topological index for $\varphi$, first note that $\omega_{\mathbb{T}^{2}}(\varphi)=\omega_{\mathbb{T}^{2}}\left(\left(z^{m} \otimes z^{n}\right) e^{\psi}\right)=\omega_{\mathbb{T}^{2}}\left(z^{m} \otimes z^{n}\right)+\omega_{\mathbb{T}^{2}}\left(e^{\psi}\right)=\omega_{\mathbb{T}^{2}}\left(z^{m} \otimes z^{n}\right)$, since $\omega_{\mathbb{T}^{2}}\left(e^{\psi}\right)=0$. Let $\omega_{1}$ be the unique topological index on $C\left(\mathbb{T}^{2}\right)$ related to the indicial triple $\Omega_{1}$ defined above. Then

$$
\begin{aligned}
\omega_{\mathbb{T}^{2}}\left(z^{m} \otimes z^{n}\right) & =\frac{1}{2} \operatorname{tr}_{\mathbb{T}^{2}}\left(\left(z^{m} \otimes z^{n}\right)^{-1} \mathrm{~d}\left(z^{m} \otimes z^{n}\right)\right) \\
& =\frac{1}{2} \operatorname{tr}_{1}\left(\left(z^{m} \otimes z^{n}\right)^{-1} \mathrm{~d}\left(z^{m} \otimes z^{n}\right)\right) \\
& =\omega_{1}\left(z^{m} \otimes z^{n}\right) \\
& =\theta \omega_{\mathbb{T}}\left(z^{m}\right)+\omega_{\mathbb{T}}\left(z^{n}\right) \quad(\text { by Theorem [2.2) } \\
& =\theta m \omega_{\mathbb{T}}(z)+n \omega_{\mathbb{T}}(z)=\theta m+n \quad(\text { by [6, Example 2.7]). }
\end{aligned}
$$

Hence, $\omega_{\mathbb{T}^{2}}(\varphi)=\theta m+n$.

Remark 3.3. It follows immediately from Proposition 3.2 that the value of the topological index $\omega_{\mathbb{T}^{2}}$ at any $\varphi \in \operatorname{Inv}\left(C\left(\mathbb{T}^{2}\right)\right)$ is independent of the choice of the character $\tau$.

Let $\Omega=(\mathcal{L}, F, \operatorname{tr})$ be an indicial triple for the $\mathrm{C}^{*}$-algebra $C$ and let $P=(F+1) / 2$. If $\varphi \in C$, then we call $T_{\varphi}=P \varphi P$ a Toeplitz element associated to $\Omega$. The $\mathrm{C}^{*-}$ algebra $\mathcal{A}$ generated by all these elements is called the Toeplitz algebra associated to $\Omega$. Note that $\mathcal{A}$ is unital with $P$ as its unit. We define the trace $\operatorname{Tr}$ on $\mathcal{A}$ as the restriction of tr to $\mathcal{A}$. An element $a \in \mathcal{A}$ is called an $\Omega$-Fredholm element if there exists an element $b \in \mathcal{A}$ such that $P-a b, P-b a \in \mathcal{M}_{\mathrm{Tr}}$. The element $b$ is called a partial inverse of $a$. The Tr-index of $a$, or the Fredholm index of $a$ relative to Tr, is defined by the relation index $\Omega(a)=\operatorname{Tr}(a b-b a)$. Moreover, $a$ is an $\Omega$-Fredholm element if and only if there exists an element $c \in \mathcal{A}$ such that $P-a c, P-c a \in \mathcal{K}_{\operatorname{Tr}}$. By [6. Theorem 3.1], if $\varphi$ is an invertible element of $C$, then $T_{\varphi}$ is an $\Omega$-Fredholm element and $\operatorname{index}_{\Omega}\left(T_{\varphi}\right)=-\omega(\varphi)$, where $\omega$ is the unique topological index related to $\Omega$. The Toeplitz algebra associated to the indicial triple $\Omega_{\mathbb{T}}$ defined above is denoted by $\mathcal{A}_{\mathbb{T}}$. If $\varphi \in C(\mathbb{T})$, then the Toeplitz element $T_{\varphi}$ is an $\Omega_{\mathbb{T}}$-Fredholm element if and only if it is a Fredholm operator in the classical sense. Moreover, in this case the $\operatorname{Tr}_{\mathbb{T}}$-index is identical to the classical Fredholm index, where $\operatorname{Tr}_{\mathbb{T}}$ is the restriction of the trace $\operatorname{tr}_{\mathbb{T}}$ to $\mathcal{A}_{\mathbb{T}}$.

Denote by $\mathcal{A}_{\mathbb{T}^{2}}$ the Toeplitz algebra associated with the indicial triple $\Omega_{\mathbb{T}^{2}}$ and denote by $\operatorname{Tr}_{\mathbb{T}^{2}}$ the restriction of the trace $\operatorname{tr}_{\mathbb{T}^{2}}$ to $\mathcal{A}_{\mathbb{T}^{2}}$. The $\mathrm{C}^{*}$-algebra $\mathcal{A}_{\mathbb{T}^{2}}$ is *-isomorphic to the Toeplitz algebra $\mathcal{T}_{\mathbb{T}^{2}}$ generated by Toeplitz operators on the quarter-plane defined in the introduction. Thus, we can identify these two $\mathrm{C}^{*}{ }_{-}$ algebras. By [6, Theorem 3.1], the following theorem is an immediate corollary of Proposition 3.2

Theorem 3.4. If $\varphi \in \operatorname{Inv}\left(C\left(\mathbb{T}^{2}\right)\right)$, then the Toeplitz element $T_{\varphi}$ associated to the indicial triple $\Omega_{\mathbb{T}^{2}}$ is an $\Omega_{\mathbb{T}^{2}}$-Fredholm element and the Fredholm index of $T_{\varphi}$ relative to $\operatorname{Tr}_{\mathbb{T}^{2}}$ is

$$
\operatorname{index} \Omega_{\mathbb{T}^{2}}\left(T_{\varphi}\right)=-\theta m-n,
$$

where $m, n$ are the unique integers such that $\varphi=\left(z^{m} \otimes z^{n}\right) e^{\psi}$, for some $\psi \in C\left(\mathbb{T}^{2}\right)$. 
The following theorem gives a characterization of $\Omega_{\mathbb{T}^{2}}$-Fredholm Toeplitz operators. Before proving it, we give some properties of the Toeplitz algebra $\mathcal{A}_{\mathbb{T}^{2}}$. By 2. Proposition 1], $\mathcal{A}_{\mathbb{T}^{2}}=\mathcal{A}_{\mathbb{T}} \otimes \mathcal{A}_{\mathbb{T}}$, where $\mathcal{A}_{\mathbb{T}}$ is the Toeplitz algebra associated to the indicial triple $\Omega_{\mathbb{T}}$. By [2, p. 205], there is a unital $*$-homomorphism $\pi_{\mathbb{T}^{2}}$ : $\mathcal{A}_{\mathbb{T}^{2}} \rightarrow C\left(\mathbb{T}^{2}\right)$ such that $\pi_{\mathbb{T}^{2}}\left(T_{\varphi}\right)=\varphi$, for all $\varphi \in C\left(\mathbb{T}^{2}\right)$, and $\operatorname{ker}\left(\pi_{\mathbb{T}^{2}}\right)=\operatorname{Com}\left(\mathcal{A}_{\mathbb{T}^{2}}\right)$. Moreover, by [2, p. 207],

$$
\operatorname{Com}\left(\mathcal{A}_{\mathbb{T}^{2}}\right)=\mathcal{A}_{\mathbb{T}} \otimes \operatorname{Com}\left(\mathcal{A}_{\mathbb{T}}\right)+\operatorname{Com}\left(\mathcal{A}_{\mathbb{T}}\right) \otimes \mathcal{A}_{\mathbb{T}}
$$

By the theory of classical Toeplitz operators, $P_{\mathbb{T}} \mathbf{K}\left(L^{2}(\mathbb{T})\right) P_{\mathbb{T}}=\operatorname{Com}\left(\mathcal{A}_{\mathbb{T}}\right)=\mathcal{K}_{\operatorname{Tr}_{\mathbb{T}}}$, where $\operatorname{Tr}_{\mathbb{T}}$ is the restriction of the $\operatorname{trace}^{\operatorname{tr}} \mathrm{tr}_{\mathbb{T}}$ to $\mathcal{A}_{\mathbb{T}}$.

Theorem 3.5. Let $\varphi \in C\left(\mathbb{T}^{2}\right)$. The Toeplitz element $T_{\varphi}$ is an $\Omega_{\mathbb{T}^{2}}$-Fredholm element if and only if $\varphi \in \operatorname{Inv}\left(C\left(\mathbb{T}^{2}\right)\right)$.

Proof. First we show that $\mathcal{K}_{\operatorname{Tr}_{\mathbb{T}^{2}}} \subseteq \operatorname{Com}\left(\mathcal{A}_{\mathbb{T}^{2}}\right)$. If $S \in \mathcal{K}_{\operatorname{Tr}_{\mathbb{T}^{2}}}$, then $S \in \mathcal{K}_{\operatorname{tr}_{\mathbb{T}^{2}}}$. We can write $S=a+b$, where $a \in \mathcal{L}_{\mathbb{T}} \otimes \mathcal{K}_{\operatorname{tr}_{\mathbb{T}}}, b \in \mathcal{K}_{\operatorname{tr}_{\mathbb{T}}} \otimes \mathcal{L}_{\mathbb{T}}$.

By density, we can choose a sequence $\left(a_{n}\right)_{n \geq 1}$ in $\mathcal{L}_{\mathbb{T}} \odot \mathcal{K}_{\operatorname{tr}_{\mathbb{T}}}$ such that $a_{n} \rightarrow a$. It can be shown that $P_{\mathbb{T}} u P_{\mathbb{T}} \in \mathcal{A}_{\mathbb{T}}$ whenever $u \in \mathcal{L}_{\mathbb{T}}$. Similarly, $P_{\mathbb{T}} v P_{\mathbb{T}} \in \mathcal{K}_{\operatorname{Tr}_{\mathbb{T}}}$ whenever $v \in \mathcal{K}_{\operatorname{tr}_{\mathbb{T}}}$. Thus, $P_{\mathbb{T}^{2}} a_{n} P_{\mathbb{T}^{2}} \in \mathcal{A}_{\mathbb{T}} \odot \mathcal{K}_{\operatorname{Tr}_{\mathbb{T}}} \subseteq \mathcal{A}_{\mathbb{T}} \otimes \mathcal{K}_{\operatorname{Tr}_{\mathbb{T}}}$, for all $n \geq 1$. Hence,

$$
P_{\mathbb{T}^{2}} a P_{\mathbb{T}^{2}} \in \mathcal{A}_{\mathbb{T}} \otimes \operatorname{Com}\left(\mathcal{A}_{\mathbb{T}}\right) \subseteq \operatorname{Com}\left(\mathcal{A}_{\mathbb{T}^{2}}\right) .
$$

By a similar argument, we can show that $P_{\mathbb{T}^{2}} b P_{\mathbb{T}^{2}} \in \operatorname{Com}\left(\mathcal{A}_{\mathbb{T}^{2}}\right)$. Therefore, $S=$ $P_{\mathbb{T}^{2}} S P_{\mathbb{T}^{2}} \in \operatorname{Com}\left(\mathcal{A}_{\mathbb{T}^{2}}\right)$. Thus, $\mathcal{K}_{\mathbb{T r}_{\mathbb{T}^{2}}} \subseteq \operatorname{Com}\left(\mathcal{A}_{\mathbb{T}^{2}}\right)=\operatorname{ker}\left(\pi_{\mathbb{T}^{2}}\right)$.

Now if $T_{\varphi}$ is an $\Omega_{\mathbb{T}^{2}}$-Fredholm element, then there is an element $S \in \mathcal{A}_{\mathbb{T}^{2}}$ such that both of the elements $P_{\mathbb{T}^{2}}-T_{\varphi} S, P_{\mathbb{T}^{2}}-S T_{\varphi}$ belong to the ideal $\mathcal{K}_{\operatorname{Tr}_{\mathbb{T}^{2}}}$. It follows immediately that $\varphi \pi_{\mathbb{T}^{2}}(S)=\pi_{\mathbb{T}^{2}}(S) \varphi=1$. Hence, $\varphi$ is an invertible function. The opposite direction follows from Theorem 3.4 .

Corollary 3.6. Let $\varphi \in C\left(\mathbb{T}^{2}\right)$. Then the Toeplitz element $T_{\varphi}$ is an $\Omega_{\mathbb{T}^{2}}$-Fredholm element with index $\Omega_{\mathbb{T}^{2}}\left(T_{\varphi}\right)=0$ if and only if $\varphi=e^{\psi}$, for some $\psi \in C\left(\mathbb{T}^{2}\right)$.

Proof. Let $\varphi \in C\left(\mathbb{T}^{2}\right)$ and let $T_{\varphi}$ be an $\Omega_{\mathbb{T}^{2}}$-Fredholm element with zero $\Omega_{\mathbb{T}^{2}}$ Fredholm index. By Theorem [3.5. $\varphi \in \operatorname{Inv}\left(C\left(\mathbb{T}^{2}\right)\right)$. Thus, $\varphi=\left(z^{m} \otimes z^{n}\right) e^{\psi}$, for a unique pair of integers $m, n$ and for some $\psi \in C\left(\mathbb{T}^{2}\right)$. By Theorem 3.4 , $-\theta m-n=\operatorname{index}_{\Omega}\left(T_{\varphi}\right)=0$. Hence, $m=n=0$, since $\theta$ is irrational. Thus, $\varphi=e^{\psi}$. The reverse implication is clear.

Now we consider the extension of our results to Toeplitz operators acting on the Hardy space $H_{N}^{2}\left(\mathbb{T}^{2}\right)=\bigoplus_{k=1}^{N} H^{2}\left(\mathbb{T}^{2}\right)$ with symbols in $C_{N}=\mathbf{M}_{N}\left(C\left(\mathbb{T}^{2}\right)\right)=$ $C\left(\mathbb{T}^{2}\right) \otimes \mathbf{M}_{N}(\mathbb{C})$. Let $\Omega_{\mathbb{T}^{2}}$ be the indicial triple for $C\left(\mathbb{T}^{2}\right)$ defined above. Define the algebra $\mathcal{L}_{N}=\mathbf{M}_{N}\left(\mathcal{L}_{\mathbb{T}^{2}}\right)=\mathcal{L}_{\mathbb{T}^{2}} \otimes \mathbf{M}_{N}(\mathbb{C})$ and define the lower semicontinuous trace $\operatorname{tr}_{N}: \mathcal{L}_{N}^{+} \rightarrow[0,+\infty]$ as $\operatorname{tr}_{N}\left(\left[a_{i j}\right]\right)=\sum_{i=1}^{N} \operatorname{tr}_{\mathbb{T}^{2}}\left(a_{i i}\right)$, for any $\left[a_{i j}\right] \in \mathcal{L}_{N}^{+}$. Let $F_{N}=$ $F_{\mathbb{T}^{2}} \otimes 1_{N}$, where $1_{N}$ is the unit in the algebra $\mathbf{M}_{N}(\mathbb{C})$. Then, by [6, Theorem 2.9], $\Omega_{N}=\left(\mathcal{L}_{N}, F_{N}, \operatorname{tr}_{N}\right)$ is an indicial triple for $C_{N}$. Moreover, if $\Phi \in \operatorname{Inv}\left(C_{N}\right)$, then $\operatorname{det} \Phi$ is a non-vanishing function on $\mathbb{T}^{2}$ and $\omega_{N}(\Phi)=\omega_{\mathbb{T}^{2}}(\operatorname{det} \Phi)=\theta m+n$, where $m, n$ are the unique integers such that $\operatorname{det} \Phi=\left(z^{m} \otimes z^{n}\right) e^{\psi}$, for some $\psi \in C\left(\mathbb{T}^{2}\right)$ and $\omega_{N}$ is the topological index related to $\Omega_{N}$. Let $\mathcal{A}_{N}$ be the Toeplitz algebra related to $\Omega_{N}$ and let $\operatorname{Tr}_{N}$ be the restriction of the $\operatorname{trace}^{\operatorname{tr}} \operatorname{tr}_{N}$ to $\mathcal{A}_{N}$. Now we prove the index theorem for Toeplitz operators with symbols belonging to $C_{N}$. 
Theorem 3.7. Let $\Phi \in C_{N}$. Then $T_{\Phi}$ is an $\Omega_{N}$-Fredholm operator if and only if $\operatorname{det} \Phi$ is non-vanishing on $\mathbb{T}^{2}$ and in this case we have

$$
\operatorname{index}_{N}\left(T_{\Phi}\right)=-\theta m-n
$$

where $m, n$ are the unique integers such that $\operatorname{det} \Phi=\left(z^{m} \otimes z^{n}\right) e^{\psi}$, for some continuous function $\psi$ on $\mathbb{T}^{2}$ and index $_{N}$ is the $\operatorname{Tr}_{N}$-index mapping.

Proof. We define the unital $*$-homomorphism $\pi_{N}: \mathcal{A}_{N} \rightarrow C_{N}$, such that $\pi_{N}(S)=$ $\left[\pi_{\mathbb{T}^{2}}\left(s_{i j}\right)\right]$, where $S=\left[s_{i j}\right] \in \mathcal{A}_{N}$. That is, $\pi_{N}$ is the inflation homomorphism of $\pi_{\mathbb{T}^{2}}$. It is easy to show that $\pi_{N}\left(T_{\Phi}\right)=\Phi$, for all $\Phi \in C_{N}$ and $\operatorname{ker}\left(\pi_{N}\right)=\mathbf{M}_{N}\left(\operatorname{ker}\left(\pi_{\mathbb{T}^{2}}\right)\right)$. By some matrix manipulations, we can show that $\mathcal{M}_{\operatorname{Tr}_{N}} \subseteq \mathbf{M}_{N}\left(\mathcal{M}_{\operatorname{Tr}^{2} 2}\right)$. Hence, by the proof of Theorem 3.5, $\mathcal{K}_{\operatorname{Tr}_{N}} \subseteq \mathbf{M}_{N}\left(\mathcal{K}_{\operatorname{Tr}_{\mathrm{T}^{2}}}\right) \subseteq \mathbf{M}_{N}\left(\operatorname{ker}\left(\pi_{\mathbb{T}^{2}}\right)\right)$.

Let $\Phi \in C_{N}$ such that $T_{\Phi}$ is an $\Omega_{N}$-Fredholm operator. Then there is $S \in \mathcal{A}_{N}$ such that $P_{N}-S T_{\Phi}, P_{N}-T_{\Phi} S \in \mathcal{K}_{\operatorname{Tr}_{N}}$. Hence, $1-\pi_{N}(S) \Phi=1-\Phi \pi_{N}(S)=0$. Thus, it follows immediately that $\operatorname{det} \Phi$ is a non-vanishing function on $\mathbb{T}^{2}$. The rest of the assertions follow immediately from [6, Theorem 3.1] and the paragraph preceding this theorem.

\section{REFERENCES}

1. C. A. Berger and L. A. Coburn, Wiener-Hopf Operators on $U_{2}$, Integr. Equ. Oper. Theory 2 (1979), 139-173. MR543881 (81c:47031)

2. R. G. Douglas and R. Howe, On the $C^{*}$-Algebra of Toeplitz Operators on the Quarter-Plane, Trans. Amer. Math. Soc. 158 (1971), no. 1, 203-217. MR0288591(44:5787)

3. G. J. Murphy, $C^{*}$-Algebras and Operator Theory, Academic Press, Boston, MA, 1990. MR.1074574 (91m:46084)

4. MR.1369029 (96m:47021)

5. - The Index Theory Associated to a Non-finite Trace on a $C^{*}$-Algebra, Canadian Math. Bull. 48 (2005), no. 2, 251-259. MR 2137103 (2006c:46058)

6. - Topological and Analytical Indices in $C^{*}$-Algebras, J. Funct. Anal. 234 (2006), 261276. MR 2216901 (2006m:46090)

7. J. Phillips and I. Raeburn, An Index Theorem for Toeplitz Operators with Noncommutative Symbol Space, J. Funct. Anal. 120 (1994), 239-263. MR.1266310 (95j:47035)

8. W. Rudin, Fourier Analysis on Groups, Interscience Publications, New York and London, 1962. MR0152834(27:2808)

9. E. van Kampen, On Almost Periodic Functions of Constant Absolute Value, J. London Math. Soc. 12 (1937), 3-6.

Department of Mathematics, Faculty of Science, The $7^{\mathrm{Th}}$ of October University, P. O. Box 2478, Misurata, Libya

E-mail address: adbabadi@yahoo.com 